\title{
Multiple Temporal and Semantic Processes During Verbal Fluency Tasks in English-Russian Bilinguals
}

Alan J. Lerner MD ${ }^{1,2, *}$, Michelle Crough ${ }^{3}$, Steven Lenio MD4, Wojbor Woyczynski ${ }^{5}$, and Frances M. Lissemore $\mathrm{PhD}^{1,2}$

${ }^{1}$ Neurology Department, Case Western Reserve University School of Medicine, Cleveland, OH, USA

${ }^{2}$ Neurology Department, University Hospitals Cleveland Medical Center, Cleveland, OH, USA

${ }^{3}$ Case Western Reserve University School of Medicine, Cleveland, $\mathrm{OH}, \mathrm{USA}$

${ }^{4}$ Department of Neurology, University of Colorado Anschutz Medical Campus, Aurora, CO, USA

${ }^{5}$ Department of Mathematics, Applied Mathematics and Statistics, and Center for Stochastic and Chaotic Processes in Science and Technology, Case Western Reserve University, Cleveland, $\mathrm{OH}, \mathrm{USA}$

*Corresponding author

Alan J. Lerner, MD

3619 Park east Drive

Beachwood, OH 44122

Alan.lerner@case.edu

Fax 12162016424

Office 12164646449

Short Title: Temporal and semantic process in Bilinguals

Keywords: semantic fluency, bilingualism, clustering, cognition, stochastic modeling 


\section{Abstract}

48 Category fluency test (CFT) performance is sensitive to cognitive processes of executive control and memory storage and access, and widely used to measure cognitive performance especially 50 in early Alzheimer's Disease. Analytical variables have included the number of items named, and various methods to identify and quantify clusters of semantically related words and cluster switches. Also encoded in the response sequence are temporal patterns as shown by "bursts" of responses and pauses between items, that have not been received attention in determining cluster characteristics.

We studied a group of 51 adult Russian-English bilinguals and compared CFT responses

based on two clustering methodologies: the semantic-based method (SEM) and a novel method based on the time interval between words (TEMP) with 8 different intercall time thresholds from

$0.25 \mathrm{sec}-15 \mathrm{sec}$. Each participant performed the task in both languages. Total number of words and cluster count was greater in Russian than English for both scoring methods, but cluster size did not differ between languages. We also studied stochastic modeling characteristics based on detrending of the "exponential exhaustion" effect seen with CFT, with most notable that total recall capacity $(\mathrm{N} \infty)$ was greater in Russian than English $(P<.05)$. Multiple demographic variables, and recent and lifetime usage of each language, affected both cognitive performance as measured by the Montreal Cognitive Assessment (MOCA; given in English only). Differential performance is driven by differences in demographics, more words stored in memory, and semantic and timing recall strategies. 


\section{Introduction}

Category fluency testing (CFT) is a measure of verbal fluency often employed in clinical and neuro-linguistic assessments. A subject is asked to name as many members of a category as possible, e.g. "animals", in a given time, generally 60 seconds. The number of responses produced on the animal naming test of category fluency is widely thought to reflect an individual's ability to produce clusters of semantically related animal names and to rapidly switch between clusters of names [1]. This ability depends on a wide range of cognitive processes including lexical access speed, executive function, education, and the size of an individual's vocabulary [2-5]. It is also well known that verbal fluency responses per unit time are subject to "exponential exhaustion" when response numbers per unit time are binned [3]; our previous work has extended these findings by use of statistical methods to detrend the data as shown by using stochastic modeling of response times between young and old subjects, and between older adults with normal cognition and those with varying degrees of cognitive impairment $[6,7]$. Thus, it appears that multiple cognitive processes are working simultaneously and contribute to the simplest output measure, the total number of words recalled in 60 seconds $\left(\mathrm{N}_{60}\right)$.

To assess clustering ability and to better understand these cognitive processes and how they contribute to semantic fluency, multiple methodologies and subject populations have been employed using semantic fluency testing [1, 8-11]. Previous studies in bilingual populations have shown small differences between languages, but have often included individuals with bilingualism of varying languages and different methods ascertaining usage between languages. Troyer et al. [1] established a method of analyzing cluster-switch data that has been widely used in studies of semantic fluency. Published studies have often relied on two raters independently assessing a sequence of responses and determining whether consecutive responses are part of the same cluster. 
Historically, cluster-switch analyses have been based on the supposition that responses

are generated in "bursts" with a pause before the respondent continues with another burst of responses, and that these bursts contain semantically related words. Combining "burstiness"

and semantic relatedness led to the prevailing notion that related items are stored in semantic

memory such that they are accessed in rapid succession. A number of studies examining the

sequence and patterns of category fluency responses have shed light on how semantic memory

is organized and accessed, but significant debate still exists over the utility of category fluency

to study semantic structure [6] [12] [13] [14] .

102

Here we report both semantic (SEM) and temporal clustering (TEMP) the response

sequences in an animal naming task in bilingual Russian-English cognitively normal adults.

104 Combined with the MOCA and demographics and response-related temporal variables gives a 105 multi-dimensional view of understanding verbal fluency output as a composite measure of 106 multiple factors.

\section{Methods}

\section{Recruitment}

The Institutional Review Board of University Hospitals Cleveland Medical Center approved this study; IRB ID \#05-13-13, and written informed consent was obtained for all participants prior to start of study procedures.

Participants were recruited from community recreation centers and residential retirement facilities known to serve populations of foreign-born citizens in suburban Cleveland, OH. All 116 procedures were approved by the University Hospitals Institutional Review Board prior to 117 recruitment. Participants were interviewed individually out of hearing range from other persons 118 in the interview area, and were compensated with a $\$ 25$ gift card. Each person informed about 119 the study was asked whether they spoke any language in addition to English. From this 
120 exchange the interviewer was able to determine whether the speaker's English was adequate

121 to participate, and if so then the person was consented out of hearing range of other persons.

122 Consent process includes questioning the potential participant to assess their understanding of

123 the study. Demographic information collected included age, place of birth, age at the time

124 participant moved to the United States, and age when participant began learning English.

125 Language dominance was self-reported by the participant.

126

127

128

129

130

131

132

133

134

135

136

137

138

139

140

141

142

143

144

145

\section{Russian and English Usage Index and Education}

We attempted to model bilingualism as a continuous variable rather than a dichotomous variable, since there is no standard quantitative threshold of "bilingualism". Participants were asked to estimate the relative use of each language by decade over their lifespan and over the past year ("recent" (English or Russian) usage). This gives a rough approximation of bilingualism as a continuous rather than a discrete variable which varies with age and life experience. We also obtained a self-report of age participant began speaking English.

Education completed was divided into five categories as follows: Less than High school graduate (1), High school graduate (2), some college (3), college graduate (4), post-graduate education (5).

\section{Testing Procedures}

The animal naming task was administered twice to each participant, once with responses in English and once with responses in Russian. Between the two trials, participants completed the Montreal Cognitive Assessment (MOCA) in English [15], and the order of languages for the CFT (English first or Russian first) was randomized to control for priming effects by the first trial of the second. 
The Montreal Cognitive Assessment (MOCA) and animal naming tests were recorded

147 using a handheld digital device. The recordings were transcribed and the time from the start of

148 the trial to the start of each word (elapsed time) was calculated using WavePad Sound Editor

149 (NCH Software Inc., Greenwood, CO).

Responses from the Russian trials were translated into English by a native Russian

151 speaker, and we recorded the total number of non-repeated responses not including errors in

15260 seconds. Two raters scored each trial for semantic clustering ("SEM" method), following the

153 Troyer et al. [1997] method with the following exceptions: we did not assign any response to

154 more than one cluster, we counted cluster size as the number of words in a cluster, and we

155 counted single words (i.e. those not semantically associated with a response preceding or

156 following) as a cluster size of one. Pearson correlation coefficient ( $r$ ) between raters for semantic

157 cluster scoring was 0.9 for the English CFT, and 0.84 for the Russian CFT.

\section{Clustering procedures: Semantic (SEM) and Time (TEMP)}

For the both scoring methods, the intercall times (time between the start of consecutive

responses) was recorded. Clusters based on time (temporal clusters or "TEMP”) were analyzed without regard to the semantic relationship among responses. In developing this new approach to clustering, it is recognized that there is no standardized intercall duration threshold known to

be optimal. If a duration shorter than the minimum was chosen, then each item would be its own cluster of a single word. At the far end, thresholds greater than the maximum intercall time up to 60 seconds would perforce result in a single cluster. Therefore, we analyzed the data using thresholds of $0.25,0.5,0.75,1,1.5,2,5$ and 15 seconds.

Mean and median cluster size (number of words in a cluster) across time duration 170 thresholds, the average cluster size ( $\mathrm{N}_{60} /$ \# clusters) were calculated for SEM scoring method, 
Additionally, we determined two additional temporal variables. The initial latency is the

duration from 0 seconds to first item named. We also calculated the duration from time at last

174 item named to 60 seconds. In previous work [7] this time correlated well with total items named.

175 Fig 1 illustrates the complex relationship of semantic versus temporal clustering for a single 176 subject.

177

178

179

180

181

182

183

184

185

186

187

188

189

190

191

192

193

194

195

196

197

198

199

200

Fig 1. Composition of time-based cluster and semantic-based clusters in an animal naming task. The first nine responses (dog through camel) constitute the only time-based cluster in this example; those same nine responses make up three semantic-based clusters (green and yellow markers). The remaining nine responses make up two additional semanticbased clusters and five un-clustered words (gray markers).

\section{Temporal Detrending Variables and Statistical Analysis}

Participant responses were analyzed using detrending procedures as described by Meyer et

al, 2012. This creates derived variables $\mathrm{N}_{60}$ (the number of words recalled), $\mathrm{N} \infty$ representing individual's "total recall capacity" allowing infinite time for recall, or the rate at which the subjects responses approach an asymptote; We must emphasize that the parameter $\mathrm{N}_{\infty}$ is called here the "total recall capacity" only figuratively, with quotation marks applied advisedly. The actual recall process cannot possibly extend its exponential behavior to infinite time as a matter of both mathematics and common sense. Accepting the unlimited exponential behavior would practically mean that after, say, one hour the individual's recall ability would be essentially zero, an obvious nonsense. So $\mathrm{N} \infty$ is just a useful parameter in the exponential exhaustion model. Tau $(\mathrm{T})$, which is the exponential time "latency" constant. The latter can be conveniently thought of as the time by which the individual reaches $\mathrm{e}^{-1}=36.8 \%$ of their "total recall capacity".

Additionally, the distribution of the detrended intercall times approximates the Weibull stretched exponential distribution and the three parameters of the distribution were calculated for each 
201 participant with sufficient responses [6]. The three components of the Weibull distribution:

202 gamma, which is related to speed of response; beta which relates to the shape of the response 203 distribution and eta, a scaling factor. Statistical analysis utilizing summary statistics, one-way 204 ANOVA, univariate Spearman correlations and non-parametric statistics were done using JMP 20514.0.

206 Results

207

208 Demographics

Table 1 shows the demographic characteristics of the subject cohort. More than $70 \%$ of

211 participants were female, ranged in age from 19 to 75 years, were well educated (86\%

212 graduated college or had a post-graduate education), and all but two were born outside the

213 United States, primarily in Russia or Ukraine. All participants spoke Russian before they spoke

214 English, and began English language instruction between the ages of 3 and 59 years. Table 1

215 shows subject demographics, MOCA scores, MOCA letter fluency word count and $\mathrm{N}_{60}$ in each

216 language. Table 1 also shows the life time and previous year index of usage of each language.

217 Table 2 shows the univariate correlation analysis showed that both Russian and English word

218 counts $\left(\mathrm{N}_{60}\right)$ correlated significantly between themselves, and were highly correlated with

219 MOCA score and MOCA letter fluency, education, lifetime Russian shown).

220

Table 1: Subject Demographics.

\begin{tabular}{|l|l|}
\hline & \\
\hline Total N =51 & Mean (SD) \\
\hline Age & $49.37(17.8)$ \\
\hline Level of education & \\
\hline Gender \%Female & $4.10(.83)$ \\
\hline Age moved to US & $73 \%$ \\
\hline Age started second language & $32.0(16.0)$ \\
\hline
\end{tabular}


MOCA (30 points maximum)

$21.4(4.8)$

MOCA Letter Fluency $\mathrm{N}$ words

11.1

$\mathrm{N}$ Responses in English $\left(\mathrm{N}_{60}\right)$

$16.18(6.9)$

$\mathrm{N}$ of Responses in Russian $\left(\mathrm{N}_{60}\right)$

$18.35(6.2)$

Lifetime English Index (0-1)

$0.21(0.16)$

$0.50(0.27)$

\section{Table 2: Univariate correlations} (Spearman's rho) of word production in each language and demographic variables and the Montreal Cognitive Assessment scores.

230

\begin{tabular}{|c|c|c|c|c|c|c|}
\hline & N60 English & Age & MOCA Score & $\begin{array}{l}\text { MOCA Letter } \\
\text { Fluency }\end{array}$ & $\begin{array}{l}\text { Lifetime } \\
\text { Russian } \\
\text { Index }\end{array}$ & $\begin{array}{l}\text { Most Recent } \\
\text { Russian Index }\end{array}$ \\
\hline $\mathrm{N}_{60}$ Russian & $0.43^{* \star}$ & -0.14 & $0.37^{* *}$ & $0.41^{* *}$ & -0.24 & 0.08 \\
\hline $\mathrm{N}_{60}$ English & & $-0.61^{* \star *}$ & $0.73^{\star * *}$ & $0.53^{\star \star \star}$ & $-0.54^{\star *}$ & -0.26 \\
\hline Age & & & $-0.62^{\star \star \star}$ & -0.17 & $0.50^{* *}$ & $0.44^{*}$ \\
\hline $\begin{array}{l}\text { MOCA Total } \\
\text { Score }\end{array}$ & & & & $0.59^{* * *}$ & $-0.48^{* *}$ & -0.24 \\
\hline $\begin{array}{l}\text { MOCA Letter } \\
\text { Fluency } \\
\text { Count }\end{array}$ & & & & & -0.28 & $0.68^{*}$ \\
\hline $\begin{array}{l}\text { Lifetime } \\
\text { Russian } \\
\text { Index }\end{array}$ & & & & & & $0.81^{* \star *}$ \\
\hline
\end{tabular}

\section{Comparing methods visually}

Fig 1 displays the outcomes of the two clustering methods for one participant's Russian responses in the CFT. The difference in the pattern of clusters between the two methods is striking, especially in the first 9 responses, which are grouped as one TEMP cluster and three SEM clusters. The remaining graphs for all participants' Russian responses and English responses are shown in S1 Fig and S2 Fig. 
There was no difference in the number of words produced in English or Russian tested

242 first versus English or Russian tested second in the CFT trials, indicating there was no priming

243 effect of repeating the test within a short time. Likewise, the order of languages in the two trials

244 (Russian first or English first) did not make a difference in the number of words produced in that 245 language.

Table 3. $\mathbf{N}_{60}$ characteristics and the lack of priming effects based on first language tested for verbal fluency output.

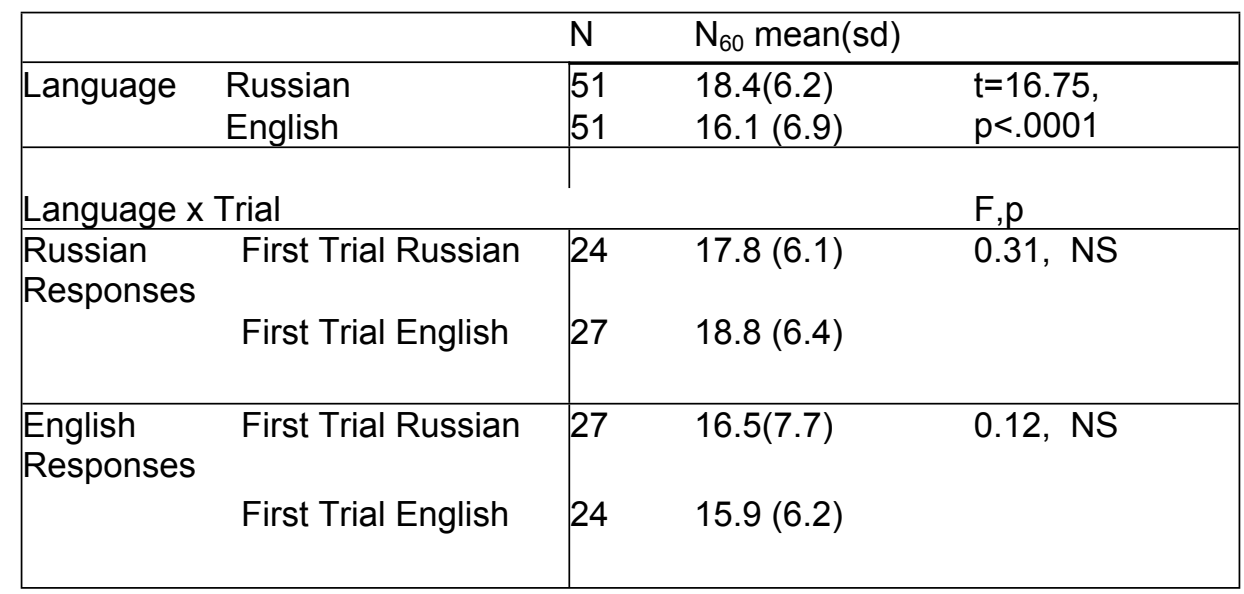

Table 4 shows the comparison between languages of the detrended response variables. $\mathrm{N} \infty$ was significantly larger in Russian, suggesting that the pool of available responses was larger, 252 and thus one factor for greater number of word responses in Russian (see table 1)

Table 4. Temporal Recall Indices in Russian and English ( $N=48)$.

\begin{tabular}{|l|l|l|l|l|l|}
\hline & No & Tau & Gamma & Beta & Eta \\
\hline Russian & $27.6(14.2)$ & $44.0(28.4)$ & $0.22(0.10)$ & $1.25(0.27)$ & $0.84(0.17)$ \\
\hline English & $22.6(10.5)$ & $43.2(46.3)$ & $0.26(0.14)$ & $1.15(0.40)$ & $0.74(0.17)$ \\
\hline $\mathrm{p}$ & $<.05$ & NS & NS & NS & $<.01$ \\
\hline
\end{tabular}


${ }^{* *}$ Values are mean(SD) English versus Russian means Compared by two tailed Wilcoxon signed rank test

Table 5 shows the univariate correlations (Spearman's rho) between word count, No, tau, average number of clusters for each clustering method, average cluster size (SEM) and median cluster size (TEMP). No correlated best with word count and clustering variables and Tau, but the correlation with temporal cluster number was not significant in Russian, while it was in English testing. Interestingly, most other variables except word count in Russian median $\mathrm{N}$ TEMP clusters were not significant.

Table 5: Univariate correlations of word count, clustering variables and detrended time variables

\begin{tabular}{|c|c|c|c|c|c|c|c|}
\hline \multirow{3}{*}{ Russian } & & $\begin{array}{l}\mathbf{N} \\
\text { SEM } \\
\text { clusters }\end{array}$ & $\begin{array}{l}\text { N Median } \\
\text { TEMP } \\
\text { clusters }\end{array}$ & \multirow{2}{*}{$\begin{array}{l}\text { Mean } \\
\text { SEM } \\
\text { Cluster } \\
\text { size } \\
0.26\end{array}$} & \multirow{2}{*}{$\begin{array}{l}\text { Median } \\
\text { TEMP } \\
\text { cluster } \\
\text { size } \\
0.56^{\star * *}\end{array}$} & \multirow{2}{*}{$\begin{array}{l}\mathbf{N} \infty \\
0.74^{* \star *}\end{array}$} & \multirow{2}{*}{$\begin{array}{l}\text { Tau } \\
0.36^{*}\end{array}$} \\
\hline & Word count $\left(\mathrm{N}_{60}\right)$ & $0.78^{\star * \star}$ & $0.75^{\star \star \star}$ & & & & \\
\hline & N Semantic Clusters & & $0.53^{* *}$ & $-0.32^{*}$ & $0.44^{\star \star}$ & $0.52^{* *}$ & 0.20 \\
\hline & Median N TEMP clusters & & & 0.14 & -0.02 & $0.67^{* * *}$ & $0.51^{* *}$ \\
\hline & Mean SEM Cluster size & & & & 0.13 & $0.38^{* *}$ & 0.27 \\
\hline & Median TEMP Cluster size & & & & & 0.26 & -0.07 \\
\hline & $\mathbf{N} \infty$ & & & & & & $0.81^{\star * *}$ \\
\hline \multirow[t]{7}{*}{ English } & Word count & $0.72^{* * *}$ & $0.83^{\star * *}$ & \multirow{4}{*}{$\begin{array}{l}0.44^{* * *} \\
-0.24 \\
0.22\end{array}$} & $0.64^{* * *}$ & $0.81^{* * *}$ & 0.04 \\
\hline & N Semantic Clusters & & $0.68^{\star \star \star}$ & & 0.31 & $0.55^{\star * *}$ & 0.12 \\
\hline & Median N TEMP clusters & & & & 0.16 & $0.63^{* * *}$ & 0.14 \\
\hline & Mean SEM Cluster size & & & & $0.44^{* *}$ & $0.43^{\star *}$ & -0.01 \\
\hline & Median TEMP Cluster size & & & & & $0.47^{* *}$ & -0.15 \\
\hline & $\mathbf{N} \infty$ & & & & & & $0.45^{\star *}$ \\
\hline & $p<.05 ;{ }^{*} p<.01 ;{ }^{* * *} p<.001$ & & & & & & \\
\hline
\end{tabular}

\section{Comparing Cluster Characteristics}


$274 \mathrm{~N}_{60}$ in Russian than English; more words generally result in more clusters. Cluster sizes,

275 however, did not differ significantly between Russian and English (Table 5). There were

276 significantly more temporal clusters in Russian than English for all durations 1 second or less.

277 However, the curves were of similar shape, and $0.25 \mathrm{sec}$ threshold duration had cluster counts

278 that approximated $\mathrm{N}_{60}$, and threshold duration of 15 seconds almost always yielded a single

279 cluster of all words (Fig 2).

280 Table 6. Clustering Characteristics by Language and Methodology of Clustering

281 (Semantic versus Temporal).

282

\begin{tabular}{|c|c|c|c|c|c|c|}
\hline & $\begin{array}{l}\mathrm{N} \\
\text { Semantic } \\
\text { Clusters }\end{array}$ & $\begin{array}{l}\text { Semantic } \\
\text { Mean } \\
\text { Cluster } \\
\text { size }\end{array}$ & \multicolumn{4}{|c|}{ Temporal Clusters (Mean (SD)) } \\
\hline & & & N Mean & $\begin{array}{l}\text { Mean } \\
\text { Cluster size }\end{array}$ & N Median & Median Cluster size \\
\hline Russian & $\begin{array}{l}9.63 \\
(3.4)\end{array}$ & $\begin{array}{l}2.09 \\
(0.6)\end{array}$ & $11.5(2.8)$ & $1.62(0.18)$ & $12.6(3.0)$ & $1.49(0.30)$ \\
\hline English & $8.1(3.3)$ & $\begin{array}{l}2.04 \\
(0.7)\end{array}$ & $\begin{array}{l}10.125 \\
(3.3)\end{array}$ & $1.53(0.20)$ & $11.2(3.6)$ & $1.39(0.29)$ \\
\hline p-value & $<.0001$ & NS & $<.01$ & $<.02$ & $<.02$ & NS \\
\hline
\end{tabular}

283

284

285

286

287

288

289

290

291

292

Fig 2: Average Number of Clusters based of Temporal Duration Thresholds by Language. 
293 The initial latency was significantly faster in Russian than English (1.14 \pm 0.58 vs. $1.94 \pm 1.70$

294 seconds; $p<0.003)$. Time from last word to 60 seconds was significantly less in Russian than

295 English (6.85 $\pm 6.1 \mathrm{sec}$ vs. $9.62 \pm 8.6 \mathrm{sec} ; \mathrm{p}<.05)$. Both of these differences probably contribute

296 to the greater number of words and ultimately clusters in Russian than English. Several

297 participants had terminal durations of more than 15 seconds, and two participants had terminal

298 durations in English of more than 30 seconds. They are also consistent with the smaller gamma

299 response variable in Russian than English, although that difference was not statistically 300 significant.

303 Discussion

Given the many analytical methods applied to CFT in the literature, it is clear that an enormous amount of information is encoded in the item content, as well as timing intervals between words. The major focus and results of this study involve comparison of the CFT in a cohort of Russian-English Bilinguals tested in both languages, and comparison of temporal and semantic cluster scoring methods using different threshold durations for defining temporal

310 clustering. Since both semantic and temporal information are simultaneously encoded in the 311 response sequence, it is important to determine their relationships on a quantitative level. Our 312 study's major findings relate to the two main aims of the study: comparison of semantic and 313 temporal processing between languages, and the feasibility of measuring temporal clustering.

314 For the former aim, participants produced more responses in Russian than English, and this 315 appears multiply determined, including demographics and differential language use, but also 316 differences in response timing, total time spent engaged in task, and size of lexicon in each 
317 language as measured by total recall capacity. For the latter aim, measuring temporal clustering

318 is quite feasible and allows comparison to the established semantic clustering method.

Temporal clustering can only be done and measured when the CFT is recorded

321 continuously, rather than the binning method used in many previous studies [3] , [16] . Use of

322 different duration thresholds to define temporal clusters showed a similar pattern of temporal

323 clusters in both languages, and a maximum number of temporal clusters occur using a 1.5-2 sec

324 cutoff of the intercall duration to separate clusters.

Previous literature has expressed concern about the subjective aspects of determining semantic clustering, although the many studies using variants of Troyer's methodology have shown differences consistent with the known neurobiology of neurodegenerative disorders such

as $A D$ [9] [17]. These concerns arise from the ambiguity involved in determining semantic

331 relatedness. Thus, a sequence of dog-cat-parrot-fish-whale could be interpreted as two clusters

332 (dog-cat-parrot (Pets); fish-whale (Marine animals), or perhaps dog-cat-parrot-fish (Pets) and 333 whale (marine mammal), or as three clusters of dog-cat (pets), parrot (bird) and fish-whale 334 (marine animals).

Long duration pauses in the response sequence are common, and there is often a

"second wind" phenomenon, with a second acceleration of responses after a long pause - in

effect, restarting the task. These longer duration pauses are problematic since they suggest 338 alterations in brain processing whose meaning is ambiguous. In the SEM method but not in the 339 TEMP method, a long pause is incorporated into the sequence of a cluster raising the question 340 of whether the respondent "intended" the responses to be semantically related. That is, long 341 durations finally producing a semantically related word, may indicate the end of one cluster, and 
342 then essentially restarting the semantic association process anew. The TEMP method more

343 accurately reflects how respondents verbalized their responses and allows the pauses

344 themselves to be utilized in analyses of the retrieval process instead of "concealing" pauses

345 within clusters as in SEM. Another point related to this is that long duration pauses or stopping

346 the response sequence early effectively turn the 60 second test into a much shorter test and

347 heavily affect the number of items produced, which is the simplest analytical method for

348 evaluating semantic processing. Also to be considered in language function is the initial latency

349 of the first word and the duration from last word to 60 second end of test. Particularly the latter

350 contributes a constraint to increasing the number of words produced, effectively shortening the

35160 second test, occasionally by as much as 30 seconds. Whether this is a motivational or

352 attentional or linguistic issue cannot be determined from the available data. The lower initial

353 latency in Russian probably was one factor contributing to increased word production in that 354 language.

Hills, et al. 2015 [18] proposed two alternative but not mutually exclusive models for

semantic memory search. Their associative model is based on "a connected sequence of related items", presumably connected by frequency of usage even if the items are not closely related semantically. Second, their categorical model relies on recalling "entire predefined categories" and choosing responses from within that group. Our data may support their associative model. Sequential responses often show little semantic relatedness, for example in Fig 1 where "pig" is

361 followed by "lion" (same TEMP cluster, different SEM cluster). Hills et al. [18] refer to "low362 similarity transitions"; our data show transitions between responses that are short in time but do 363 not necessarily have "short" semantic connections. That observation supports the idea that 364 TEMP clusters correspond to the associative model because high-usage responses are likely to 365 show up in the same cluster even if they are not closely related semantically. Hills et al. conclude 366 that retrieval from semantic memory is a process both of frequency of usage and of categorical 
367 similarity, and long pauses between semantically related responses (as seen in our data)

368 support that idea. Other forms of semantic clustering using different word retrieval constructs

369 have been compared by Abenwender, Swan, Bowerman and Connolly [19] but further

370 discussion is beyond the scope of this study.

Another methodological concern of both methods involves the treatment of single word

"clusters". By definition, the first word produced is part of a cluster, and if the subsequent

response exceeded the temporal threshold, a cluster of a single word is generated. Thus, even

the definition of "temporal cluster" contains some ambiguity. Whereas semantic clusters are

derived from two independent raters, definition of temporal clustering is done automatically, but

cluster numbers vary depending on the threshold duration chosen.

Troyer et al 1997 [1] used number of switches between clusters as a proxy for direct

cluster count, stating "(s)witches were calculated as the number of transitions between clusters,

including single words....; i.e. single word clusters. Dramatic differences in count caused by

cluster definition has consequences in neuro-linguistic assessments insofar as cluster count is

considered a reflection of cognitive function. Haugrud et al. [9] note "small changes in scoring...

can change... measures of clustering, (hence) average cluster size might not be the most

effective method for differentiating AD from healthy aging" [20]. A one-word cluster has no

semantic association with a word preceding or following it, and it often exceeds in time an

association with adjacent responses. Therefore, it is also possible that single word clusters do

not totally fit SEM or TEMP criteria, and that analyses of clusters $>1$ word alone may point

towards more meaningful conclusions regarding semantic memory structure and access.

The cultural, educational and life experiences of the respondent may influence how

390 frequently words are used and how readily they are retrieved from memory, and similarly, those 391 of the rater may influence cluster composition. Interestingly, we found a lower correlation 
392 between our raters (U.S.-born native English speakers) in semantic category scoring in Russian

393 (after translation) (Pearson $r=.84$ ) than in English (Pearson $r=.90)$. This suggests it was more

394 difficult for raters to consistently infer the response relationships intended by respondents in the 395 language not spoken by our raters.

396 Temporal clustering methods may have a theoretical advantage over semantic methods

397 in terms of understanding neural function as reflected by advanced statistical methods. The 398 process of semantic recall for related terms is similar to how animals search for food in resource 399 patches (for example bees in a flower patch), which is the focus of optimal foraging theory (OFT) 400 [21]. Optimal foraging models posit that animals search patches such that foraging efficiency is 401 maximized. Likewise, "patches" of semantic memory are searched for unique animal names in 402 the CFT. OFT has recently been applied to analyses of response sequences in CFTs [18] , 403 divergent thinking processes [22], and to predict intercall times [23]. In addition to optimal 404 foraging, other kinds of models have been utilized to further understand memory association and retrieval, including mathematical, physical, and computer-based analyses [24] [25] [26] [27]

Limitations of the present study include the absence of monolingual control groups in each language, and of a general vocabulary assessment in either language before testing. Our study population, however, was highly educated (Table1) which likely indicates strong 410 vocabulary ability. The sample of participants is a convenience sample, and may not reflect the 411 wide population of Russian-English or other bilingual combinations. Application to aging and 412 disease models also awaits further study.

413 Our prior work examined CFT in groups with varying levels of cognitive impairment [7] 414 focused on intracluster and intercluster timings but did not include analysis of temporal 415 clustering. In that study, cluster size did not vary significantly across groups, but cluster counts 416 did, a pattern similar to what we found in the current analysis. Clusters with more words means 
417 fewer clusters (i.e. fewer cluster switches), and cluster switching has been used as a proxy for

418 executive function [8] [28] [29] [5]. We could likely expect that other types of category fluency,

419 e.g. vegetables, four legged animals, food or clothing, could be analyzed by the TEMP method 420 as well.

421 Category fluency testing is a clinically useful measure because of the enormous amount

422 of encoded information utilizing multiple brain processes contributing to its outcome. Integrative

423 tests, such as gait timing, clock drawing or CFT are useful screening tools precisely because

424 performance integrity implies intact brain processing, and conversely, it is sensitive to many

425 types of baseline neurological ability, brain injury and cognitive decline beyond Alzheimer's 426 disease and related disorders [30] [31]. As reviewed here, clustering reflects multiple brain 427 processes, and both semantic and temporal clustering provide insights into these very brain 428 processes. Temporally-based cluster scoring method for the animal naming task is equally 429 feasible and possibly less ambiguous than the semantic-based method although the optimal 430 threshold duration between items, used to define "clusters" is varies between respondents. 431 Additionally, temporal clustering may reduce basic inter-rater reliability because the start of a 432 cluster and its end are quantitatively determined, obviating semantic relatedness judgements. 433 This method also allows for a faster scoring process which can be easily adapted to automated 434 programming. Future studies in different populations are needed to define the relative 435 contributions of the two methods in determining the clinical and research significance of each.

\section{References}

439 1. Troyer AK, Moscovitch M, Winocur G. Clustering and switching as two components of

440 verbal fluency: evidence from younger and older healthy adults. Neuropsychology.

$441 \quad 1997 ; 11(1): 138-46$. 
442 2. Luo L, Luk G, Bialystok E. Effect of language proficiency and executive control on

443 verbal fluency performance in bilinguals. Cognition. 2010;114(1):29-41.

444 3. Rohrer D, Wixted JT, Salmon DP, Butters N. Retrieval from semantic memory and its

445 implications for Alzheimer's disease. J Exp Psychol Learn Mem Cogn. 1995;21(5):1127-39.

446 4. Shao Z, Janse E, Visser K, Meyer AS. What do verbal fluency tasks measure?

447 Predictors of verbal fluency performance in older adults. Front Psychol. 2014;5:772.

448 5. Whiteside DM, Kealey T, Semla M, Luu H, Rice L, Basso MR, et al. Verbal Fluency:

449 Language or Executive Function Measure? Appl Neuropsychol Adult. 2016;23(1):29-34.

450 6. Meyer DJ, Messer J, Singh T, Thomas PJ, Woyczynski WA, Kaye J, et al. Random local

451 temporal structure of category fluency responses. J Comput Neurosci. 2012;32(2):213-31.

452 7. Lenio S, Lissemore FM, Sajatovic M, Smyth KA, Tatsuoka C, Woyczynski WA, et al.

453 Detrending Changes the Temporal Dynamics of a Semantic Fluency Task. Front Aging

454 Neurosci. 2016;8:252.

455 8. Bertola L, Cunha Lima ML, Romano-Silva MA, de Moraes EN, Diniz BS, Malloy-Diniz

456 LF. Impaired generation of new subcategories and switching in a semantic verbal fluency test

457 in older adults with mild cognitive impairment. Front Aging Neurosci. 2014;6:141.

458 9. Haugrud N, Crossley M, Vrbancic M. Clustering and switching strategies during verbal

459 fluency performance differentiate Alzheimer's disease and healthy aging. J Int Neuropsychol

$460 \quad$ Soc. $2011 ; 17(6): 1153-7$.

461 10. Gold CA, Marchant NL, Koutstaal W, Schacter DL, Budson AE. Conceptual fluency at

462 test shifts recognition response bias in Alzheimer's disease: implications for increased false

463 recognition. Neuropsychologia. 2007;45(12):2791-801.

464 11. Weakley A, Schmitter-Edgecombe M. Analysis of verbal fluency ability in Alzheimer's

465 disease: the role of clustering, switching and semantic proximities. Arch Clin Neuropsychol.

466 2014;29(3):256-68. 
467 12. Sung K, Gordon B, Yang S, Schretlen DJ. Evidence of semantic clustering in letter-cued

468 word retrieval. J Clin Exp Neuropsychol. 2013;35(10):1015-23.

469 13. Verheyen S, Voorspoels W, Longenecker J, Weinberger DR, Elvevåg B, Storms G.

470 Invalid assumptions in clustering analyses of category fluency data: Reply to Sung, Gordon

471 and Schretlen (2015). Cortex. 2016;75:255-9.

472 14. Voorspoels W, Storms G, Longenecker J, Verheyen S, Weinberger DR, Elvevåg B.

473 Deriving semantic structure from category fluency: clustering techniques and their pitfalls.

474 Cortex. 2014;55:130-47.

475 15. Nasreddine ZS, Phillips NA, Bédirian V, Charbonneau S, Whitehead V, Collin I, et al.

476 The Montreal Cognitive Assessment, MoCA: a brief screening tool for mild cognitive

477 impairment. J Am Geriatr Soc. 2005;53(4):695-9.

478 16. Friesen DC, Luo L, Luk G, Bialystok E. Proficiency and Control in Verbal Fluency

479 Performance across the Lifespan for Monolinguals and Bilinguals. Lang Cogn Neurosci.

$480 \quad 2015 ; 30(3): 238-50$.

481 17. Raoux N, Amieva H, Le Goff M, Auriacombe S, Carcaillon L, Letenneur L, et al.

482 Clustering and switching processes in semantic verbal fluency in the course of Alzheimer's

483 disease subjects: results from the PAQUID longitudinal study. Cortex. 2008;44(9):1188-96.

484 18. Hills TT, Todd PM, Jones MN. Foraging in Semantic Fields: How We Search Through

485 Memory. Top Cogn Sci. 2015;7(3):513-34.

486 19. Abwender DA, Swan JG, Bowerman JT, Connolly SW. Qualitative Analysis of Verbal

487 Fluency Output: Review and Comparison of Several Scoring Methods. Assessment.

$488 \quad 2001 ; 8(3): 323-38$.

489 20. Lanting S, Haugrud N, Crossley M. The effect of age and sex on clustering and

490 switching during speeded verbal fluency tasks. J Int Neuropsychol Soc. 2009;15(2):196-204. 
491 21. Charnov EL. Optimal foraging, the marginal value theorem. Theor Popul Biol.

$492 \quad 1976 ; 9(2): 129-36$.

493 22. Hass RW. Semantic search during divergent thinking. Cognition. 2017;166:344-57.

494 23. Montez P, Thompson G, Kello CT. The Role of Semantic Clustering in Optimal Memory

495 Foraging. Cogn Sci. 2015;39(8):1925-39.

496 24. Queau P-Y, Woyczynski WA, Lerner AJ. Coalescing stochastic 'processes in retrival

497 from semantic memory. Mathematica Applicanda. 2015;43:187-224.

498 25. Hopfield JJ, Brody CD. What is a moment? "Cortical" sensory integration over a brief

499 interval. Proc Natl Acad Sci U S A. 2000;97(25):13919-24.

500 26. Tapson JC, Cohen GK, Afshar S, Stiefel KM, Buskila Y, Wang RM, et al. Synthesis of 501 neural networks for spatio-temporal spike pattern recognition and processing. Front Neurosci.

$502 \quad 2013 ; 7: 153$

503 27. Zak SH, Upatising V, Hui S. Solving linear programming problems with neural networks:

504 a comparative study. IEEE Trans Neural Netw. 1995;6(1):94-104.

505 28. Carter SF, Caine D, Burns A, Herholz K, Lambon Ralph MA. Staging of the cognitive 506 decline in Alzheimer's disease: insights from a detailed neuropsychological investigation of 507 mild cognitive impairment and mild Alzheimer's disease. Int J Geriatr Psychiatry. $508 \quad 2012 ; 27(4): 423-32$.

509 29. Kirova AM, Bays RB, Lagalwar S. Working memory and executive function decline 510 across normal aging, mild cognitive impairment, and Alzheimer's disease. Biomed Res Int.

$511 \quad 2015 ; 2015: 748212$.

512 30. Pastor-Cerezuela G, Fernández-Andrés MI, Feo-Álvarez M, González-Sala F. Semantic

513 Verbal Fluency in Children with and without Autism Spectrum Disorder: Relationship with

514 Chronological Age and IQ. Front Psychol. 2016;7:921. 
515 31. Schmidt CSM, Nitschke K, Bormann T, Römer P, Kümmerer D, Martin M, et al.

516 Dissociating frontal and temporal correlates of phonological and semantic fluency in a large

517 sample of left hemisphere stroke patients. Neuroimage Clin. 2019;23:101840.

519 Supporting Information

S1 Fig. Semantic and temporal cluster compositions for responses in Russian in the

521 animal naming task. Response count is graphed over time (60 seconds), and accompanying chart shows assignment of semantic clusters and of temporal clusters. The top line of the chart identifies participant ID number and response count, with sequence of animal names in the second line. Cluster switches are indicated by alternate shading of boxes below the response sequence (semantic clusters-- third line; temporal clusters-- fourth line); no shading under a word 526 indicates it was not part of a cluster.

S2 Fig. Semantic and temporal cluster compositions for responses in English in the

animal naming task. Response count is graphed over time (60 seconds), and accompanying chart shows assignment of semantic clusters and of temporal clusters. The top line of the chart

530 identifies participant ID number and response count, with sequence of animal names in the 531 second line. Cluster switches are indicated by alternate shading of boxes below the response sequence (semantic clusters-- third line; temporal clusters-- fourth line); no shading under a word 533 indicates it was not part of a cluster.

\section{Acknowledgements}

535 This study was funded by the Brain Health and Memory Center, Neurological Institute of 536 University Hospitals Cleveland Medical Center. The authors wish to thank S. Klayman for 537 translating responses from Russian. 
bioRxiv preprint doi: https://doi.org/10.1101/2020.12.14.422659; this version posted December 14,2020 . The copyright holder for this preprint (which was not certified by peer review) is the author/funder, who has granted bioRxiv a license to display the preprint in perpetuity. It is made available under aCC-BY 4.0 International license.

538

539 Funding

540 This study was supported by the Brain Health and Memory Center at University Hospitals

541 Cleveland Medical Center and NIA P30 AG062428 (AJL,FML). 
bioRxiv preprint doi: https://doi.org/10.1101/2020.12.14.422659; this version posted December 14, 2020. The copyright holder for this preprint (which was not certified by peer review) is the author/funder, who has granted bioRxiv a license to display the preprint in perpetuity. It is made available under aCC-BY 4.0 International license.

Average Temporal Cluster Number

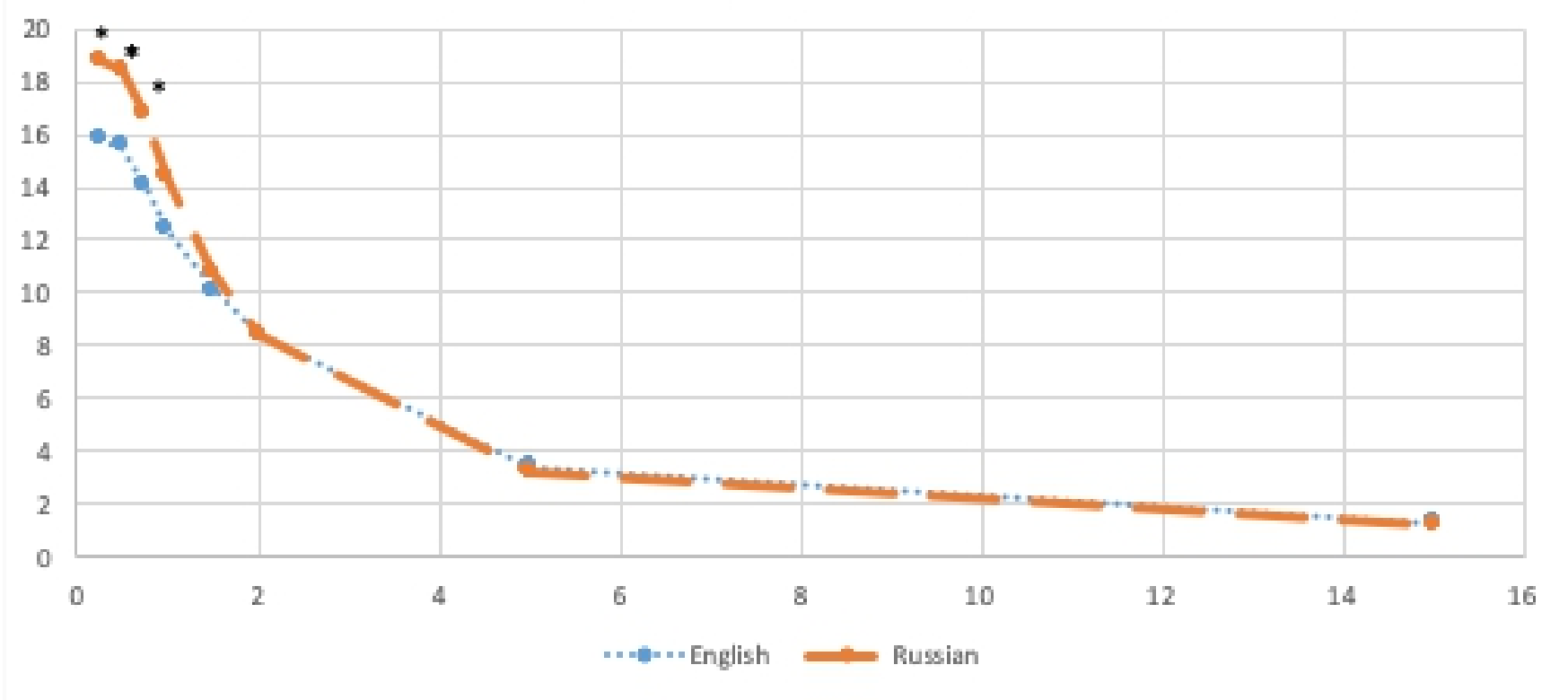


